\title{
What's new? What's certain? - Scoring Search Results in the Presence of Overlapping Data Sources
}

\author{
Philipp Hussels, Silke Trißl, and Ulf Leser \\ Humboldt-Universität zu Berlin, Institute of Computer Sciences, D-10099 Berlin, \\ Germany \\ \{hussels, trissl, leser\}@informatik.hu-berlin.de
}

\begin{abstract}
Data integration projects in the life sciences often gather data on a particular subject from multiple sources. Some of these sources overlap to a certain degree. Therefore, integrated search results may be supported by one, few, or all data sources. To reflect these differences, results should be ranked according to the number of data sources that support them. How such a ranking should look like is not clear per se. Either, results supported by only few sources are ranked high because this information is potentially new, or such results are ranked low because the strength of evidence supporting them is limited.

We present two scoring schemes to rank search results in the integrated protein annotation database Columba. We define a surprisingness score, preferring results supported by few sources, and a confidence score, preferring frequently encountered information. Unlike many other scoring schemes our proposal is purely data-driven and does not require users to specify preferences among sources. Both scores take the concrete overlaps of data sources into account and do not presume statistical independence. We show how our schemes have been implemented efficiently using SQL.
\end{abstract}

\section{Introduction}

In research on molecular biology, very often knowledge from different domains is needed to answer practical questions. Imagine a researcher asking for the three-dimensional structure of a protein that participates in a certain metabolic pathway and is associated with a certain disease. This researcher has to query multiple data sources. For instance, she could access the Protein Data Bank (PDB) [1] for the protein structure, KEGG [7] for pathway information, and PubMed to find information about protein-disease associations. However, for the latter two aspects many other data sources could be used as well.

We call those different aspects of biomedical objects a domain. For a protein such domains are 3D structure, sequence, fold, functional classification, other proteins it interacts with, processes it is involved in, diseases it is associated with, etc. For many domains there exist multiple sources. For example, information about pathways can be found in KEGG [7], aMAZE [9], Reactome [6], and several other resources. These resources usually overlap extensionally, i.e., 


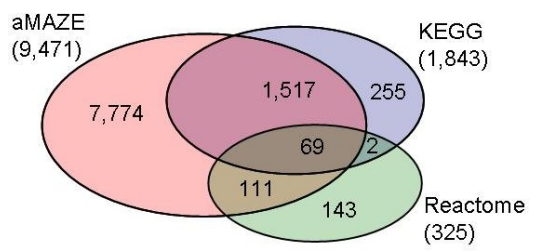

Fig. 1. The figures in brackets state the total number of enzyme-enzyme pairs that are connected through a chain of reaction $\rightarrow$ substrate $\rightarrow$ reaction. The figures in the different partitions show the number of pairs that occur in only one, two, or all three data sources.

store the same information (and not only the same kind of information) because they partially draw their content from the same data sets. For three sources on pathways the overlap can be seen in Figure 1. But the sources also contain different and potentially unique information, due to different methodology used to curate, integrate, select, or produce the data. Thus, when a researcher looks for the pathways a given protein is involved in, the results may vary considerably depending on the chosen source. We want to provide users with a ranking of search results depending on the particular set of data sources that support it.

\subsection{Data Model}

We assume that a user is interested in information about a particular class of biological entities, called the primary domain $P$. Objects in $P$ are described by objects in other data sources. A group of data sources that contain information about the same type of entities or even the same entities is called secondary domain $D_{i}$. The content of secondary domains is comprised of data from various data sources $S_{i 1}, \ldots, S_{i m}$ and link sources $R_{i 1}, \ldots, R_{i l}$, where $i$ is the secondary domain. If the domain is clear $i$ can be omitted.

The link sources $R_{1}, \ldots, R_{l}$ contain entries $(s, p)$ with $s \in S_{i}$ and $p \in P$, i.e., they provide links between objects in data sources of a secondary domain and objects in the primary domain. Thus, every object in a data source of domain $D_{i}$ is linked through link sources to one or more objects in $P$ and vice versa. We also say that an object in $P$ is annotated by objects in data sources of $D_{i}$. This situation is depicted in Figure 2.

A query selects entries from $P$ by setting conditions on annotations in different domains. The result of a query, written as $\operatorname{res}(q)$ is the set of objects in $P$ that comply with these conditions through at least one data and one link source for every domain mentioned in $q$. For a single result $p \in \operatorname{res}(q)$ we say that the result is supported by at least one qualified annotation in every secondary domain. As the data and link sources in a domain overlap, an annotation supporting a result may stem from different data sources and may be linked by different link sources. According to the degree of dependence between the data and link sources of a domain, certain combinations of sources frequently support 


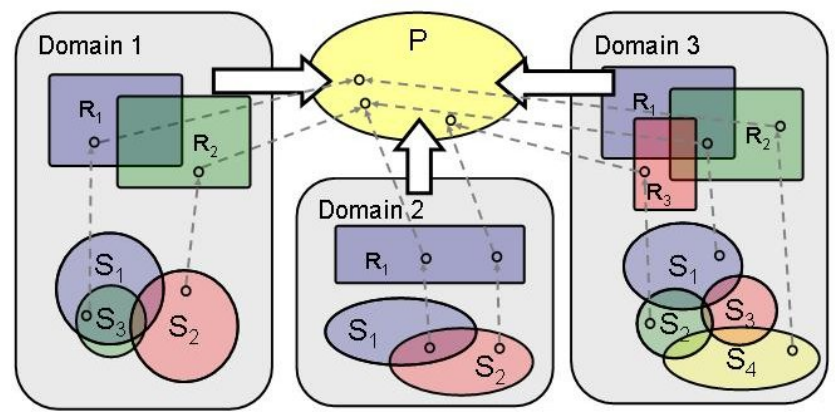

Fig. 2. The objects in the primary domain $P$ are annotated by objects from three secondary domains $\left(D_{1} \ldots D_{3}\right)$. Every domain contains several secondary data and link sources.

query results, while other combination of sources rarely do. We make use of this fact to assign query results scores for confidence and surprisingness.

\subsection{Scoring of Results}

This paper is about ranking results in a setting described above. Integrating many sources instead of manually selecting some (the 'best' ones) comes at the risk of large result sets. Therefore, ranking of results becomes important. However, ranking is not a one-dimensional problem. Clearly, a user is most confident in results supported by all data sources. In the previous example a result that is supported by KEGG, aMAZE, and Reactome is one where users will be most confident that it is biologically true. However, such results are sort-of common place and thus potentially boring. Some researcher might be more interested in the contrary, i.e., surprising results supported by only few sources. For example, a result supported by Reactome but not by aMAZE is rather unexpected, because Reactome is much smaller. Thus, a-priori chances to find a result supported only by Reactome are small. If this occurs it makes a good starting point for a more thorough investigation with a higher chance to produce some new findings.

Both scores, confidence and surprisingness, are important. It depends on the concrete application which ranking scheme should be used for a search. In this paper we present a method to compute both scores for integrated search results over multiple domains where each domain is formed from multiple data and link sources. In contrast to much of the related work, our method does not require expert knowledge, but is merely based on the properties of the data sources themselves, i.e., the overlaps between them. 


\subsection{Paper outline}

The paper is structured as follows. We discuss related work in the next section. The surprisingness score is defined in Section 3 and the confidence score in Section 4. In Section 5 we show how to expand both measures to multidomain queries. Section 6 describes the application of the scores in the integrated database Columba. Section 7 shows how to implement the scoring scheme and also gives some experimental results. Section 8 concludes the paper.

\section{Related work}

One option to use information from different data sources is to provide the user only with information supported by all selected data sources, i.e., the information a user is most confident in. Marcotte et al. [10] proposed such a method for the reconstruction of metabolic pathways from protein-protein interaction data. Clearly, their results are highly trustworthy, but a biologically correct proteinprotein interaction supported only by some data sources will not be considered. In contrast Yanai \& DeLisi [17] used a union of different interaction data sources. This leads to good coverage, as all known interactions are listed, but possibly also many incorrect protein-protein interactions are included.

The problem of giving the user all possible information ordered according to some criteria is addressed by many projects. Internet search engines rank hits according to their expected usefulness for the query. The protein-protein database STRING [12] integrates information on protein-protein interactions from different data sources such as high-throughput experiments, literature search, or sequence comparison. A confidence score for every object is created. This score is either uniform within a data source, e.g., for an integrated source without further knowledge, or individual for every object, e.g., when text mining methods are used to extract protein-protein interactions from publications. Similar methods have been described in the area of functional analysis of microarray experiments [5]. A general framework for specifying and using such quality scores for query optimization and result ranking has been proposed in [13]. All these methods build on expert knowledge about the data sources. Such ratings are highly subjective and not easy to obtain.

In this paper we propose a method that ranks results without the need for expert knowledge. A similar idea was proposed by Florescu et al. in [4] for the purpose of query optimization. Given a query they want to optimize the ratio between the execution cost and the size of the result set. To answer the query they first estimate which sources will return most results and then choose $k$ sources, based on the selectivity of the source and the overlap with other sources.

A different approach is described by Lacroix et al. in [8] for estimating the size of the result set. They assume a network of interlinked sources and data objects. A query poses conditions on a start source and returns results from a

primary source by analyzing all paths from the start to the primary source. To estimate the size of the result set they pre-compute overlap statistics for different 
paths using sampling. In Bleiholder et al. [2] these overlap statistics are used to optimize queries over multiple data sources to solve the Budgeted Maximum Coverage problem. In contrast to this work, we use a simpler model (primary and secondary sources) and focus on ranking of results in result sets, not on query optimization.

\section{Surprisingness of Results}

We now present a framework for measuring the surprisingness of a search result. Confidence will be defined in Section 4. We develop our model starting from a single domain with a single data and link source and then extend it to multiple data and link sources. The extension to multi-domain queries is given in Section 5.

We assume that the result set contains objects from the primary source. The user can restrict this set by setting conditions on objects in secondary domains. An object in the primary source is contained in the result set if it is supported by at least one qualified annotation in every queried domain.

\subsection{Single Data Source}

We start with the simple scenario of a single domain $D$, a single data source $S$, and a single link source $R$ as shown in Figure 3(a). Without loss of generality we assume that every annotation $s \in S$ is linked to at least one object $p \in P$ through at least on link $r \in R$ (we can safely delete all other annotations and links since they can never select entries in $P$ ). A query selects objects in $D$ and determines the set of objects in $P$ that are linked by at least one link in $R$.

For a given query $q$ we calculate the probability that a randomly chosen object $p \in P$ is part of the result set of $q$. We first derive the a-priori probability that a randomly chosen annotation $s \in S$ is linked to a randomly chosen object $p \in P$ :

$$
P((s, p) \in R)=\frac{|R|}{|P \times S|}=\frac{|R|}{|P| *|S|}
$$

A randomly chosen $p \in P$ takes part in the query result if it is linked to at least one qualified annotation $s \in S$ by at least one link $r \in R$. If we assume that $q$ selects $k$ annotations and take into account that a single object in $P$ can be selected by multiple annotations in $S$, then the probability that a concrete $p \in \operatorname{res}(q)$ is selected is precisely the probability that not none of the $k$ selected annotations is linked to $p$, which gives:

$$
P(p \in \operatorname{res}(q))=1-\left(1-\frac{|R|}{|P| *|S|}\right)^{k}
$$

Clearly, we could also estimate the value of $k$ a-priori using attribute selectivities. Note that this formula ranks all objects in a result set of a query equal. 
This is expected, as we want to rank a result by the subset of sources that supports it in any domain. Therefore, differences in the computed score only appear when more than one source is present.

\subsection{Multiple Sources in a Single Domain}

We now extend our framework to the case of $m$ data sources $S_{i}$ and $l$ link sources $R_{j}, 1 \leq i \leq m$ and $1 \leq j \leq l$ for a single domain $D$ as shown in Figure 3(b).

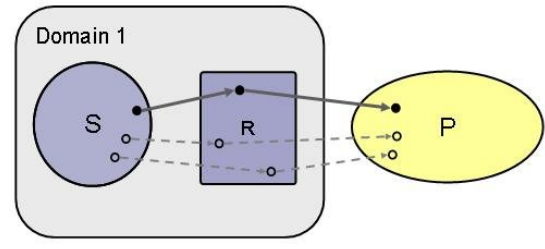

(a) Single data and link source.

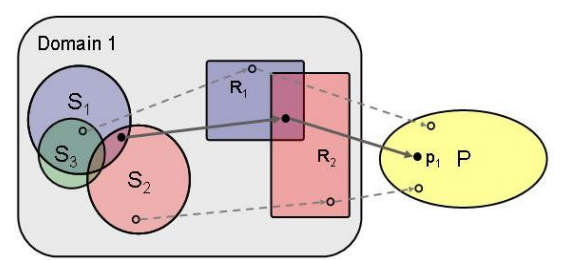

(b) Multiple data and link sources.

Fig. 3. An object $p \in P$ is supported by objects $s \in S_{i}$ in a domain.

An object $p \in P$ is in the result set of a given query $q$ if it is supported by at least one qualified annotation $s$ linked through at least one link $r$. However, $s$ as well as $r$ can be contained in various combination of sources. Consider the situation shown in Figure 3(b) with three overlapping data sources $S_{1}, S_{2}$, and $S_{3}$ and two overlapping link sources $R_{1}$ and $R_{2} . S_{1}$ and $S_{3}$ strongly overlap, while $S_{2}$ mostly contains divergent data. In this situation it is likely that a query result is linked to a qualified annotation contained in $\left(S_{1} \cap S_{3}\right) \backslash S_{2}$ or $S_{2} \backslash\left(S_{1} \cup S_{3}\right)$. Such query results shall be assigned a low score for surprisingness. We would rate a result more unlikely and therefore more surprising that is supported by a qualified annotation in $S_{3} \backslash\left(S_{1} \cup S_{2}\right)$. Clearly, to compute the score we also have to consider over which combination of link sources $s$ is linked to $p$. Note that according to our understanding of surprisingness, a high score might also be assigned to results with incorrect annotations. This is in the line of our argument, since errors can be considered surprising and certainly require user attention.

The space of all annotations in $D$ is partitioned into disjoint subsets according to the overlaps of data and link sources. Some of these subsets are represented by different colors in Figure 3(b). We call these partitions in data sources $Z_{1}, \ldots, Z_{n}$. The assignment of annotations to partitions can be represented by a domainvector $v$ of size $m * l$ for a domain with $m$ data and $l$ link sources. If annotation $s \in S_{i}$ and $(s, p) \in R_{j}$ we set $v_{i, j}=1$, and $v_{i, j}=0$ otherwise. In Figure 3(b) an annotation contained in $S_{1} \cap S_{3} \backslash S_{2}$ that is linked over $R_{1}$ corresponds to the domain-vector $v_{i, 1}=101$ and $v_{i, 2}=000$. It follows that $2^{m * l}$ different domain vectors are possible. Now consider a single annotation $s$ selected by $q$. Intuitively, a $p$ linked to $s$ is the more surprising, the smaller the partition $Z_{k}$ is in which $s$ lies. 
However, we need some more work to derive a suitable definition for surprisingness. We compute the surprisingness for each annotation selected by a query which might later be aggregated into a score for an object $p$ linked to multiple annotations. Let $Z_{k}$ be the partition in which an annotation $s$ lies that is selected by a query $q$. We estimate the probability that $p$ is verified by all sources that contain $Z_{k}$ and no others, which depends on the a-priori overlaps of sources. That means, we want to know how likely it is that a result for a given query is verified by a certain combination of available sources. The less likely, the more surprising is the result.

To answer this we first estimate the probability that for a given query a result is verified by a particular data source $S_{x}$ provided that it is verified by at least one source in $D$. This is different from Equation 2 because $p$ can be selected by other sources than $S_{x}$. Let $q_{S x}$ denote the subset of res $(q)$ that is verified by $S_{x}$. Using Bayes's Theorem we get:

$$
P\left(p \in q_{S x} \mid p \in \operatorname{res}(q)\right)=\frac{P\left(p \in \operatorname{res}(q) \mid p \in q_{S x}\right) * P\left(p \in q_{S x}\right)}{P(p \in \operatorname{res}(q))}
$$

Clearly, the probability that an object is verified by at least one data source provided that it is verified by a particular $S_{x}, P\left(p \in \operatorname{res}(q) \mid p \in q_{S x}\right)$, is 1 because the first event logically implies the second one. The a-priori probability $P(p \in \operatorname{res}(q))$ is given by Equation 2, where $|S|$ now denotes the set of all unique annotations in $D$. We only miss the a-priori probability $P\left(p \in q_{S x}\right)$. For this probability we must take into account that not every object in $S$ is contained in $S_{x}$ and not every link in $R$ links annotations $s \in S_{x}$ to a $p \in P$. We therefore can identify a subset of $R$, denoted as $R_{x}$, that only contains links from $s \in S_{x}$. Analogously, we can distinguish a subset of $P$, called $P_{x}$ that contains entries $p$ that are supported by an annotation $s \in S_{x}$.

$$
\begin{aligned}
P\left(p \in q_{S x}\right) & =1-\left(1-\frac{\left|P_{x}\right|}{|P|} * \frac{\left|S_{x}\right|}{|S|} * \frac{\left|R_{x}\right|}{\left|P_{x}\right| *\left|S_{x}\right|}\right)^{k} \\
& =1-\left(1-\frac{\left|R_{x}\right|}{|P| *|S|}\right)^{k}
\end{aligned}
$$

Thus, Equation 3 can be rewritten as:

$$
P\left(p \in q_{S x} \mid p \in \operatorname{res}(q)\right)=\frac{1-\left(1-\frac{\left|R_{x}\right|}{|P| *|S|}\right)^{k}}{1-\left(1-\frac{|R|}{|P| *|S|}\right)^{k}}
$$

We now determine the probability that a particular $p$ is supported by qualified annotations in a partition $Z_{k}$. Here as well we denote the subset of $\operatorname{res}(q)$ verified by annotations in $Z_{k}$ as $q_{Z k}$. This gives: 


$$
P\left(p \in q_{Z k} \mid p \in \operatorname{res}(q)\right)=\frac{1-\left(1-\frac{\left|\bigcap_{S_{i} \supseteq Z_{k}} R_{i} \backslash \bigcup_{S_{i} \supseteq Z_{k}} R_{i}\right|}{|P| *|S|}\right)^{k}}{1-\left(1-\frac{|R|}{|P| *|S|}\right)^{k}}
$$

\subsection{Surprisingness Score of a Single Annotation}

To value the surprisingness of a single annotation we use the measure of selfinformation as defined by Shannon. Consider a domain-vector $v$ as a symbol in a message, the self-information of $v, I(v)$, depends on the probability of its occurrence and is defined as $I(v)=-\log _{2}(P(v))$. Accordingly, we calculate the surprisingness score for $p$ that is contained in the result set of a given query by applying the probability that $p$ is supported by an $s \in Z_{k}$ using Equation 7 . Definition 1 formalizes this approach:

Definition 1 (Surprisingness for a single annotation) Let $q$ be a query selecting an annotation $s$, let $p$ be linked to $s$, and let $s$ lie in partition $Z_{k}$ of $D$. The surprisingness $S(p, s)$ of $p$ with respect to $s$ is defined as:

$$
S(p, s)=-\log _{2} P\left(p \in q_{Z k} \mid p \in \operatorname{res}(q)\right)
$$

\subsection{Surprisingness Score for a Single Domain}

Equation 7 only gives the probability that a given $p$ is linked to a given annotation $s$ selected by a query $q$. But we want a score for $p$ given all its linked annotations selected by $q$, as shown in Figure 4 . Therefore, we will need to aggregate scores of multiple $s$.

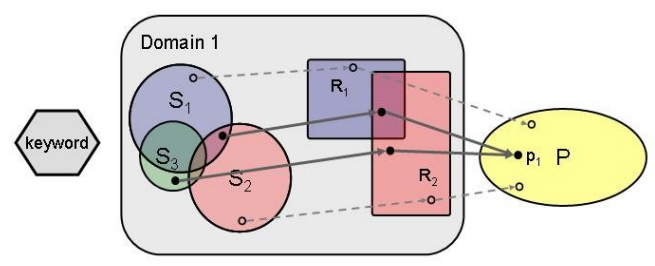

Fig. 4. An object linked to two qualified annotations.

Suppose, the primary domain contains protein structures and the secondary domain multiple data sources about scientific publications. Assume we query with a keyword and receive a structure $p$ that is linked to multiple qualified publications. If all publications are contained in the same combination of data sources, intuitively the number of publications that verify $p$ does not influence 
its surprisingness. In this case $p$ shall be assigned the same surprisingness score as assigned to a single publication. Now imagine $p$ is linked to multiple qualified publications contained in different combinations of data sources as depicted in Figure 4. Clearly, if most selected publications linked to $p$ are highly surprising we also want to assign $p$ a high surprisingness score. We therefore define the surprisingness of $p$ as the average of the surprisingness scores for every qualified publication that supports $p$.

Definition 2 (Surprisingness for multiple annotations) Let $q$ be a query and $p \in \operatorname{res}(q)$ be linked to a set $T$ of annotations selected by $q$. The surprisingness $S(p, T)$ of $p$ is defined as:

$$
S(p, T)=\frac{1}{|T|} \sum_{s \in T} S(p, s)
$$

\section{Confidence of Results}

As explained in Section 1, researches are not solely interested in highly surprising query results but also in trustworthy results. A researcher might want to rank those results high that are likely to be correct. Having multiple data sources in a domain, intuitively every data source that verifies a query result $p$ increases the confidence in the correctness of $p$. Thus, a straightforward method to value the confidence of a query result would be to count the number of sources verifying the result. But here too we have to consider that the different data sources within a domain are not independent. If, for example, a query result $p$ is verified by two data sources, the confidence in $p$ being correct is the higher the lower the degree of dependence between those data sources is, because then it is more likely that information contained in both sources is the outcome of independent experiments rather than information stemming from the same resource.

Consider again the situation shown in Figure 3(b). We are most confident in annotations that are contained in $S_{1} \cap S_{2} \cap S_{3}$ and linked through both link sources $R_{1}$ and $R_{2}$. If we consider annotations $s_{1} \in\left(S_{1} \cap S_{3}\right) \backslash S_{2}$ and $s_{2} \in\left(S_{1} \cap\right.$ $\left.S_{2}\right) \backslash S_{3}$, both linked only over $R_{1}$, we intuitively assign $s_{2}$ a higher confidence score because $S_{1}$ and $S_{3}$ strongly overlap, while $S_{1}$ and $S_{2}$ do not. More generally, for the confidence score we want to use the probability that an annotation is contained in a combination of sources given that the annotation is contained in at least one source.

Definition 3 (Confidence for a single annotation) Let $q$ be a query selecting an annotation $s$, let $p$ be linked to $s$ through $r$, and let $s$ be contained in the the partition $Z_{k}$. The confidence $C(p, s)$ of $p$ with respect to $s$ is defined as:

$$
C(p, s)=1-\log _{2} \frac{\left|\bigcap_{S_{i} \supseteq Z_{k}} S_{i}\right|}{\left|\bigcup_{S_{i} \supseteq Z_{k}} S_{i}\right|}
$$


Resulting from Definition 3 the score for a $p$ linked to an annotation $s$ that is contained in only one source is 1 . Note, the confidence score for $p_{1}$ that is annotated by $s_{1} \in Z_{k}$ is always lower or equal to the score for $p_{2}$ annotated by $s_{2} \in Z_{l}$, with $Z_{l}$ being the intersection between data sources in $Z_{k}$ and an additional data source $S_{i}$.

So far, we considered the confidence for a result supported by only a single annotation. We shall now show how to aggregate confidence scores for multiple annotations. While the number of qualified annotations linked to a query result $p$ does not influence its score for surprisingness, it clearly enhances the trust in the correctness of $p$. As we consider every single annotation as an evidence that $p$ is an answer to a given query we sum up the confidence scores of all qualified annotations linked to $p$ to calculate the confidence score of $p$.

Definition 4 (Confidence for multiple annotations) Let $q$ be a query and $p \in \operatorname{res}(q)$ be linked to a set $T$ of annotations selected by $q$. The confidence $C(p, T)$ of $p$ is defined as:

$$
C(p, T)=\sum_{s \in T} C(p, s)
$$

\section{Multi-domain Query Results}

In the last two sections we defined scores for surprisingness and confidence for single domain queries. In this section we explain how to use these values to compute a surprisingness and confidence score in a multi-domain setting.

We assume that different secondary domains are statistically independent. We can make that assumption as according to our model we group data sources that contain information about the same type of biological entities in one domain. To compute an overall surprisingness score we add up scores from all secondary domains given in a query. We do this as we consider a result surprising when it is surprising for at least one domain. In contrast, for the confidence, only those results of multi-domain queries shall be ranked high that have high confidence scores in many domains. To ensure this, we normalize single domain scores resulting from Equation 10 before multiplying all scores for the multi-domain confidence score.

\section{Multi-domain Setting: Columba}

In this section we introduce our real world example, where the scores presented in this paper are beneficial. We developed the integrated database Columba [14]. This database focuses on protein structures from the Protein Data Bank (PDB) [1] that are annotated by objects of different domains, such as fold, sequence, function, publication, metabolic pathway, or taxonomic classification.

We apply our scoring methods for ranking query results to parts of the Columba database. We use as primary domain the protein structures given by 


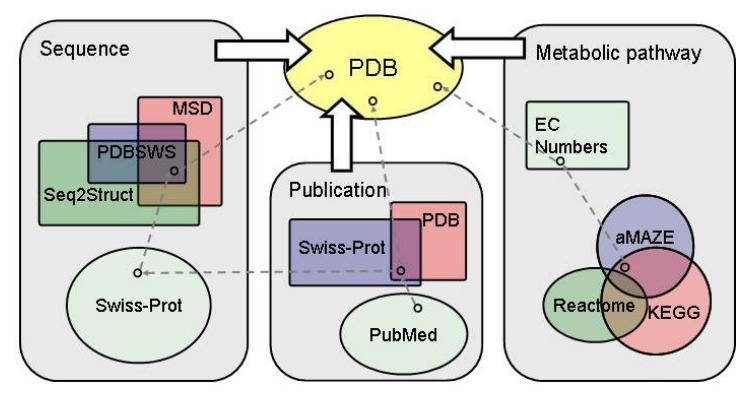

Fig. 5. The PDB annotated by secondary domains that contain multiple data sources. Note, the size and overlap of data and link sources does not necessarily reflect reality.

the PDB. Objects in the PDB are annotated by the secondary domains sequence, publication, and metabolic pathway as shown in Figure 5.

The domain sequence contains the data source Swiss-Prot [3] and has three different link sources linking entries from Swiss-Prot to entries in the PDB, namely PDBSWS [11], Seq2Struct [16], and MSD [15]. The overlap of link sources is given by identical entries in the sources.

Data sources for the domain metabolic pathway are KEGG [7], aMAZE [9], and Reactome [6]. To compare these heterogeneous data sources we extract information on the level of reactions. We store enzyme-enzyme pairs that are connected through a path enzyme $\rightarrow$ reaction $\rightarrow$ substrate $\rightarrow$ reaction $\rightarrow$ enzyme. The overlap of data sources is given by identical enzyme-enzyme pairs. If we consider enzymes as nodes and a pair of enzymes as edges in a graph we can compute paths between enzymes. We therefore can answer queries such as "Which PDB entries are less than 3 steps away from an enzyme with EC number 2.7.1.1 (Hexokinase)". The data sources are linked to the PDB through EC numbers given in the PDB as well as in the three data sources.

In the third domain publications we use PubMed as data source. The articles referenced in PubMed can be linked directly to the PDB using the references given in the PDB. But articles in PubMed can also be linked to the PDB via Swiss-Prot.

A multi-domain query for this setting is for example "Give me all protein structures that are up to 7 steps away from an enzyme with EC number 1.14.16.1 (Phenylalanine hydroxylase), linked to entries in Swiss-Prot that contain the keyword Phenylalanine catabolism, and linked to publications that mention the disease Phenylketonuria". This query returns in total 17 PDB chains. Using our scoring scheme we can rank the results according to their surprisingness and their confidence. 


\section{Implementation and Evaluation}

In this section we show which values can be precomputed and how to implement this computation inside a relational database environment. We additionally show for some exemplary queries the impact of surprisingness and confidence scores.

\subsection{Precomputation of Values}

To compute the surprisingness of an object $S(p, T)$ we must first compute for every object in a domain the probability $P\left(p \in q_{Z k} \mid p \in r e s(q)\right)$ as given by Equation 6. We therefore require information about the size of the primary domain and the size of data and link sources in secondary domains. To gather $|P|$ we simply count the number of objects in the primary domain. To gather $|S|$ and $|R|$ we first have to integrate all objects of the data and link sources of $D_{i}$ and then count the number of unique objects in both integrated sources. Knowing these values we can compute the value $c_{1}=1-\frac{|R|}{|P| *|S|}$ for domain $D_{i}$. But we also require the size of different partitions of data and link sources. To gather these data we precompute and store the domain-vector $v$ for every unique object in $S$ of dimension $D_{i}$. We can determine the size for every partition $Z_{k}$ by determining the frequency of different patterns in $v$, denoted as freq $(v)$. But to solve Equation 6 we require the value for the size of partitions in the link sources, denoted as link_size $(v)$. We can determine $l i n k \_s i z e(v)$ by summing up for every $s \in Z_{k}$ the number of $(s, p) \in R$. Knowing this value we can compute the value $c_{2}=1-\frac{\operatorname{lin} k_{-} s i z e(v)}{|P| *|S|}$. The only value in Equation 6 that can not be precomputed is $k$, the number of qualified objects in $D_{i}$. But we can substitute variables in Equation 6 to gain the following equation for $S(p, s)$ :

$$
S(p, s)=\frac{1-\left(c_{2}\right)^{k}}{1-\left(c_{1}\right)^{k}}
$$

To compute the confidence score for a result $p$ we must compute $C(p, s)$ as given by Equation 9. Here we require the size of all unions and intersections of data sources $S_{i}$ that contain $s$. Both values are independent of a particular query and therefore can be precomputed using freq $(v)$. For a given domain-vector $v$ of length $n$ the sum of freq $\left(v^{\prime}\right)$ with $v^{\prime}: v^{\prime} \wedge v=v$ is the size of the intersection and the sum of $\operatorname{freq}\left(v^{\prime \prime}\right)$ with $v^{\prime \prime}: v^{\prime \prime} \wedge v \neq 0^{n}$ is the size of the union for a combination of sources. We can thus write Equation 9 as:

$$
C(p, s)=1-\frac{\sum_{v^{\prime} \wedge v=v} \operatorname{freq}\left(v^{\prime}\right)}{\sum_{v^{\prime \prime} \wedge v \neq 0^{n}} \operatorname{freq}\left(v^{\prime \prime}\right)}
$$

We can precompute the confidence score $C(p, s)$ for all possible intersections of sources in $D_{i}$, but we have to store $2^{m * l}$ confidence values for one domain with $m$ data and $l$ link sources. This means, we can precompute the size of partitions and unions only for a limited number of data and link sources. But we expect that in real world applications such as Columba this will not be a problem. If the problem arises, some heuristics for precomputation must be introduced. 


\subsection{Implementation}

Precomputation The integrated database Columba is implemented on PostGreSQL 8.2. In Columba every data and every link source is stored in its own table. For every domain we store the domain-vectors $v$ in a separate table. We precompute and store all sizes and frequencies mentioned in the last section in statistics tables. To compute freq $\left(v^{\prime}\right)$ and $f r e q\left(v^{\prime \prime}\right)$ we use the provided functions bit_and() and bit_or() of PostGreSQL.

Execution of Queries We now describe how to use the precomputed values to compile a ranked result set for a given query. The compilation of the result set with scores is done in four steps. For every domain given in the query we first select all annotations $s$ fulfilling the conditions posed in the query and link them to entries in $P$. In this step we also return the precomputed values for every pair $(p, s)$, including $C(p, s)$. In the second step we determine $k$ and calculate $S(p, s)$. In the third step we aggregate the surprisingness and confidence scores of a single domain for an object $p$. In the last step - if the query poses conditions on multiple domains - we aggregate the scores for an object $p$ over all domains.

We will explain this by a simple example that selects chains of protein structures from the PDB that are supported by entries in Swiss-Prot, which contain the keyword Phenylalanine catabolism. Figure 6 shows the SQL query to find all combinations of PDB chains and qualified entries in Swiss-Prot. For every combination we return the values for $c_{1}$ and $c_{2}$ and the confidence score $C(p, s)$. In the next step we determine $k$ by counting all unique Swiss-Prot ids and then compute $S(p, s)$. In the last step we aggregate the scores for every PDB chain by averaging over the surprisingness scores and sum over the confidence scores.

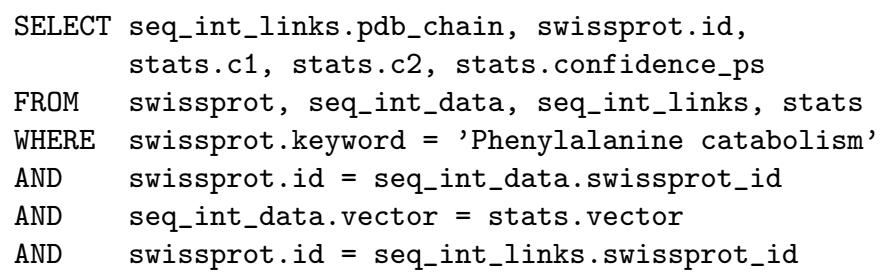

Fig. 6. SQL query to return all PDB chain - Swiss-Prot id combinations given the keyword Phenylalanine catabolism and some constants.

\subsection{Evaluation}

Overlap of Sources in Columba The three data sources that link Swiss-Prot entries to chains in the PDB have an overlap of 51,051, i.e., most of the links of MSD (total 69,785) and PDBSWS (total 69,303) are contained in that overlap (data not shown). Seq2Struct contains in total 216,539 links, i.e., most links between the PDB and Swiss-Prot are only contained in that source. The overlap 
for the data sources of metabolic networks is given in Figure 1. aMAZE contains the highest number of enzyme-enzyme combinations, mainly due to the fact that reactions in aMAZE are always bi-directional. In the publications domain we have 73,945 links from PDB chains directly to PubMed, most of which are contained in the 223,156 links over Swiss-Prot to PubMed.

Queries on Columba To evaluate our approach we queried the Columba database using keywords on a single domain, e.g., "Give me all PDB chains annotated by Swiss-Prot entries that contain the keyword Phenylalanine catabolism". We used all distinct keywords from Swiss-Prot (in total 881) to query the sequence domain and 1,000 randomly chosen MeSH terms to query the publication domain. For evaluation we excluded empty result sets and result sets in which all entries had the same confidence or surprisingness score. This results in 727 result sets for the sequence domain and 695 for the publication domain.

For every result set we normalized the confidence and surprisingness scores to gain values between 0 and 1 . We sorted entries in the result set into 11 buckets $([0,0.1),[0,1,0.2), \ldots$, and an own bucket for $[1])$ according to their confidence or surprisingness scores. Figures $7(\mathrm{a})-(\mathrm{d})$ show the average frequencies of entries in a bucket for the result sets of the sequence and publication domain.

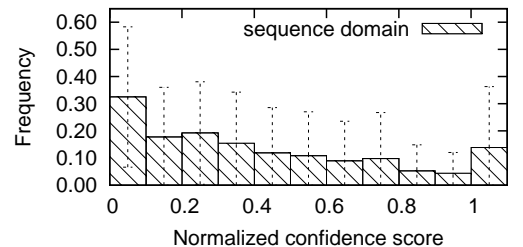

(a) Confidence - sequence domain.

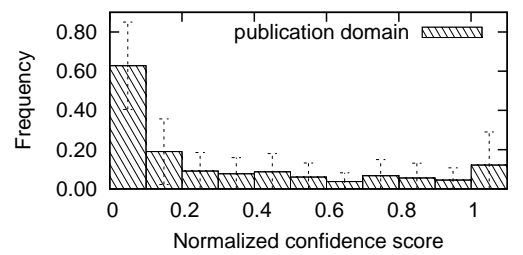

(c) Confidence - publication domain.

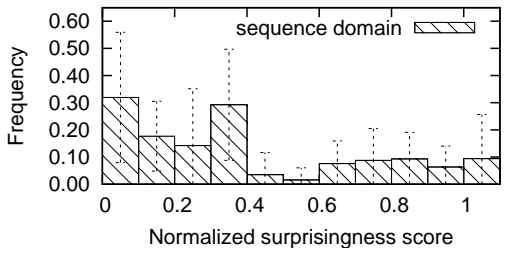

(b) Surprisingness - sequence domain.

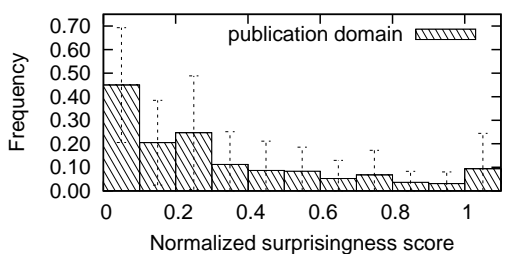

(d) Surprisingness - publication domain.

Fig. 7. Average frequency and standard deviation of normalized confidence and surprisingness scores for queries on the sequence and the publication domain.

Figures $7(\mathrm{a})$ and (c) show that on average only $14 \%$ of the entries in a result set for the sequence and $12 \%$ for the publication domain have a normalized confidence value of 1 . Most entries in the result set $50 \%$ for sequence and $82 \%$ for publication domain) have normalized confidence values between 0 and 0.2 . Figures 7(b) and (d) show the data for the surprisingness score. In both domains 
on average about $9 \%$ of entries in result sets have a normalized surprisingness score of 1 . Here as well the largest bucket is the bucket that contains entries with scores between 0 and 0.1. The high standard deviation for all buckets can be explained by varying distributions of scores within the result sets. Consider a result set in which the entries only have two different scores, which is typical for small result sets. Clearly, a subset of entries will be in the bucket with value 1 , while the other subset is in one of the remaining 10 buckets. This subset can contain one entry or all but one entry of the result set. The figures for the metabolic pathway domain are not displayed. Concluding, the figures indicate that both scores will nicely rank entries in the result set for the given domains.

We now present an exemplary query on multiple domains and parts of its result set. The query "Give me all PDB chains that are annotated by SwissProt entries with the keyword Glycolysis, that are linked to PubMed articles containing the word Glycolysis, and that are at most three steps away from the protein with EC number 2.7.1.1 (Hexokinase)" returns 109 chains from the PDB. Table 1(a) and 1(b) show the top 5 results sorted either by confidence or surprisingness.

Table 1. The top 5 query results for different sorting.

(a) Sorted by Confidence

\begin{tabular}{cc|cc}
$\begin{array}{c}\text { PDB } \\
\text { id }\end{array}$ & chain & Confidence & $\begin{array}{c}\text { Surprising- } \\
\text { ness }\end{array}$ \\
\hline 1dqr & A & 1.0 & 20.6 \\
1dqr & B & 1.0 & 20.6 \\
1g98 & A & 0.6 & 18.3 \\
1g98 & B & 0.6 & 18.3 \\
1xtb & A & 0.6 & 18.3 \\
\hline
\end{tabular}

(b) Sorted by Surprisingness

\begin{tabular}{cc|cr}
$\begin{array}{c}\text { PDB } \\
\text { id }\end{array}$ & chain & Confidence & $\begin{array}{c}\text { Surprising- } \\
\text { ness }\end{array}$ \\
\hline 1pky & C & 0.1 & 22.8 \\
2pgi & - & 0.5 & 22.2 \\
1c7q & A & 0.5 & 22.2 \\
1c7r & A & 0.5 & 22.2 \\
1i33 & D & 0.1 & 22.2 \\
\hline
\end{tabular}

The most confident results are structures for the protein phosphoglucose isomerase. This is expected as the protein is only one reaction away from the hexokinase in the glycolysis pathway. Note, the top 5 most surprising results contain completely different chains in the PDB, including a pyruvate kinase (1pky) that is also in the glycolysis pathway, but further away from hexokinase than phosphoglucose isomerase.

\section{Conclusion}

In this paper we defined the surprisingness and the confidence score for an object in a result set that is annotated by multiple, possibly overlapping data sources. Both scores can be used to rank objects in a result set. We showed its applicability to biological data using parts of the integrated database Columba. In the future we plan to integrate both scoring schemes in the Columba web interface. In addition we will further investigate the possibility to extend both score 
definitions to also account for the distribution of source combinations within a single result set.

\section{References}

1. Berman, H., Westbrook, J., Feng, Z., Gilliland, G., et al. The Protein Data Bank. Nucleic Acids Research, 28(1):235-242, Jan 2000.

2. Bleiholder, J., Khuller, S., Naumann, F., Raschid, L., and Wu, Y. Query Planning in the Presence of Overlapping Sources. In Proceedings of the EDBT, pages 811828, 2006. Springer.

3. Boeckmann, B., Bairoch, A., Apweiler, R., Blatter M.-C., et al. The SWISSPROT protein knowledgebase and its supplement TrEMBL in 2003. Nucleic Acids Research, 31(1):365-370, Jan 2003.

4. Florescu, D., Koller, D., and Levy, A. Using Probabilistic Information in Data Integration. In Proceedings of the VLDB, pages 216-225, 1997. Morgan Kaufmann.

5. Huttenhower C., Hibbs, M., Myers, C., and Troyanskaya, O. A scalable method for integration and functional analysis of multiple microarray datasets. Bioinformatics, 22(23):2890-2897, Dec 2006.

6. Joshi-Tope, G., Gillespie, M., Vastrik, I., D'Eustachio, P., et al. Reactome: a knowledgebase of biological pathways. Nucleic Acids Research, 33:D428-D432, Jan 2005.

7. Kanehisa, M., Goto, S., Kawashima, S., and Nakaya, A. The KEGG databases at GenomeNet. Nucleic Acids Research, 30:42-46, Jan 2002.

8. Lacroix, Z., Murthy, H., Naumann F., and Raschid, L. Links and Paths through Life Sciences Data Sources. In Procceding of the DILS in Lecture Notes in Computer Science, volume 2994, pages 203-211, 2004. Springer.

9. Lemer, C., Antezana, E., Couche, F. and Fays, F., et al. The aMAZE LightBench: a web interface to a relational database of cellular processes. Nucleic Acids Research, 32:D443-D448, Jan 2004.

10. Marcotte, E., Pellegrini, M., Ng, H., Rice, D., et al. Detecting protein function and protein-protein interactions from genome sequences. Science, 285(5428):751-753, Jul 1999.

11. Martin A. C. Mapping PDB chains to UniProtKB entries. Bioinformatics, 21(23):4297-4301, Dec 2005.

12. von Mering, C., Jensen, L., Snel, B., Hooper, S., et al. STRING: known and predicted protein-protein associations, integrated and transferred across organisms. Nucleic Acids Research, 33:D433-D437, Jan 2005.

13. Naumann, F., Leser, U., Freytag, J.-C. Quality-driven Integration of Heterogenous Information Systems. In Proceedings of the VLDB, pages 447-458, 1999. Morgan Kaufmann.

14. Rother, K., Müller, H., Trissl, S., Koch, I., et al. Columba: Multidimensional Data Integration of Protein Annotations. In Procceding of the DILS in Lecture Notes in Computer Science, volume 2994, pages 156-171, 2004. Springer.

15. Velankar, S., Mcneil, P., Mittard-Runte, V., Suarez, A., et al. E-MSD: an integrated data resource for bioinformatics. Nucleic Acids Research, 33:D262+, Jan 2005.

16. Via, A., Zanzoni, A., and Helmer-Citterich, M. Seq2Struct: a resource for establishing sequence-structure links. Bioinformatics, 21(4):551-553, Sep 2004.

17. Yanai, I. and DeLisi, C. The society of genes: networks of functional links between genes from comparative genomics. Genome Biology, 3(11):research0064, Oct 2002. 